\title{
Near infrared (NIR) imaging techniques using lasers and nonlinear crystal optical parametric oscillator/amplifier (OPO/OPA) imaging and transferred electron (TE) photocathode image intensifiers
}

\author{
George J. Yates and Thomas E. McDonald Jr., \\ Los Alamos National Laboratory, MS H803, Los Alamos, New Mexico \\ David E. Bliss, Stewart M. Cameron, Kenneth Greives, and Fred J. Zutavern, \\ Sandia National Laboratories, MS 1188, Albuquerque, New Mexico
}

\begin{abstract}
Laboratory experiments utilizing different near-infrared (NIR) sensitive imaging techniques for LADAR range gated imaging at "eye-safe" wavelengths are presented. An OPO/OPA configuration incorporating a nonlinear crystal for wavelength conversion of 1.56 micron "probe" or "broadcast" laser light to $807 \mathrm{~nm}$ light by utilizing a second "pump" laser at $532 \mathrm{~nm}$ for gating and gain, was evaluated for sensitivity, resolution, and general image quality. These data are presented with similar test results obtained from an image intensifier based upon a "transferred electron" (TE) photocathode with high quantum efficiency (QE) in the 1-2 micron range, with a P-20 phosphor output screen. Data presented include range-gated imaging performance in a cloud chamber with varying optical attenuation of laser reflectance images.
\end{abstract}

Keywords: Transferred electron photocathode, wavelength conversion, nonlinear crystal, OPO/OPA

\section{INTRODUCTION}

Los Alamos National Laboratory has developed a high-speed fast-shuttered intensified digital CCD camera for range-gated imaging applications. The camera, designated GY-11, is based upon a gated image intensifier fiber optically coupled to a CCD capable of continuous high repetition rate framing. Intensifier shutter times as short as 100 ps and frame periods as short as 500 microseconds have been measured for the GY-11 camera. The fast gates are from "stripline" geometry microchannel plate image intensifier (MCPII) designs. When used in conjunction with a co-located pulsed "probe" or "broadcast" laser illuminator, the intensifier gates are strategically time-phased to open in coincidence with the return beam corresponding to reflectance images from the laser striking various objects or targets at the range of interest. For use in military LADAR applications the laser must be invisible to maintain covert operation and also to provide "eye safe" wavelengths to safeguard personnel. The GY-11 camera (ref 1 ) is a visible-range imaging system which has shown good range imagery (ref 2,3 ), but at $400-800 \mathrm{~nm}$. To meet the requirement for "eye safe" imaging at 1-2 microns, Los Alamos National Laboratory and Sandia National Laboratories have collaborated on infrared (IR) and near infrared (NIR) experiments to study the merits of two technologies capable of NIR and IR imaging. These are the Transferred Electron (TE) photocathode image intensifiers manufactured by Intevac EO Sensors Division, San Jose, California, (ref 4,5) and an OPO/OPA system (ref 6) using nonlinear optical crystals. The experiments were primarily "proof of principle" laboratory experiments designed to demonstrate feasibility of NIR and IR gated imaging in scattering environments and to measure such basic parameters as the light flux and sensor responsivity required for threshold images and the resolution obtainable by the two approaches. Previous independent works utilizing nonlinear optics (ref 6,7,8,9) by both Laboratories provided useful baseline information for these collaborative experiments.

\section{TE PHOTOCATHODE CHARACTERIZATION}

The Intevac model 21D5 proximity focused planar diode image intensifier with TE photocathode has manufacturer-advertised spectral sensitivity approaching $5-20 \%$ QE from $900 \mathrm{~nm}$ to 1.8 microns. We measured the intensifier sensitivity using two methods. First we measured sensitivity at discrete wavelengths within the spectral range of the photocathode using two different pulsed lasers. Next, we used 


\section{DISCLAIMER}

This report was prepared as an account of work sponsored by an agency of the United States Government. Neither the United States Government nor any agency thereof, nor any of their employees, make any warranty, express or implied, or assumes any legal liability or responsibility for the accuracy, completeness, or usefulness of any information, apparatus, product, or process disclosed, or represents that its use would not infringe privately owned rights. Reference herein to any specific commercial product, process, or service by trade name, trademark, manufacturer, or otherwise does not necessarily constitute or imply its endorsement, recommendation, or favoring by the United States Government or any agency thereof. The views and opinions of authors expressed herein do not necessarily state or reflect those of the United States Government or any agency thereof. 


\section{DISCLAIMER}

Portions of this document may be illegible in electronic image products. Images are produced from the best available original document. 
Black Body radiation to excite the photocathode, and calculated the photon flux integral over the TE photocathode spectral range.

Our first laser experiments used a pulsed laser, Big Sky model ULTRASHG10, which provided pulses of approximately $10 \mathrm{~ns} F W H M$, and approximately $8 \mathrm{~mJ}$ energy per pulse at $1572 \mathrm{~nm}$. Our earliest tests imaged the laser light unto the Intevac intensifier photocathode and the phosphor image was fiber optically coupled to an RS-170 EEV CCD camera which was recorded with a Big Sky Beamcode data acquisition/display system. These tests yielded inconclusive results. The TE photocathode, although extremely sensitive, has a limited dynamic range is also extremely limited in available photoelectrons, therefore, operation at reduced input flux levels was required. An additional planar diode image intensifier was coupled to the Intevac intensifier to amplify its weak phosphor signals before recording with a low noise cooled slow scan CCD camera/data acquisition system, Pixel Vision model SVGF10KS. The experiment setup is shown in figure 1 . The resolution was obtained from the CCD camera line-scan profiles along the horizontal plane of an Air Force resolution pattern, which indicated coherent transfer function (CTF) of $50 \%$ of DC response at 4 line pairs $/ \mathrm{mm}$ and approximately $25 \%$ at 8 line pairs $/ \mathrm{mm}$. Other profiles indicated limiting resolution at about 12 line pairs $/ \mathrm{mm}$. Similar CTF was measured in the vertical direction. For the sensitivity data, a Laser Precision model RJ7620 radiometer was placed in the same location corresponding to the focal plane of the Intevac intensifier's photocathode. Values measured were limited to the nanojoule range only, due to the insensitivity of the radiometer in this experiment geometry. Subsequent laser experiments described later provided higher effective sensitivity for the flux energy measurements to calibrate the Intevac "transferred electron" photocathode (TEPC) response.

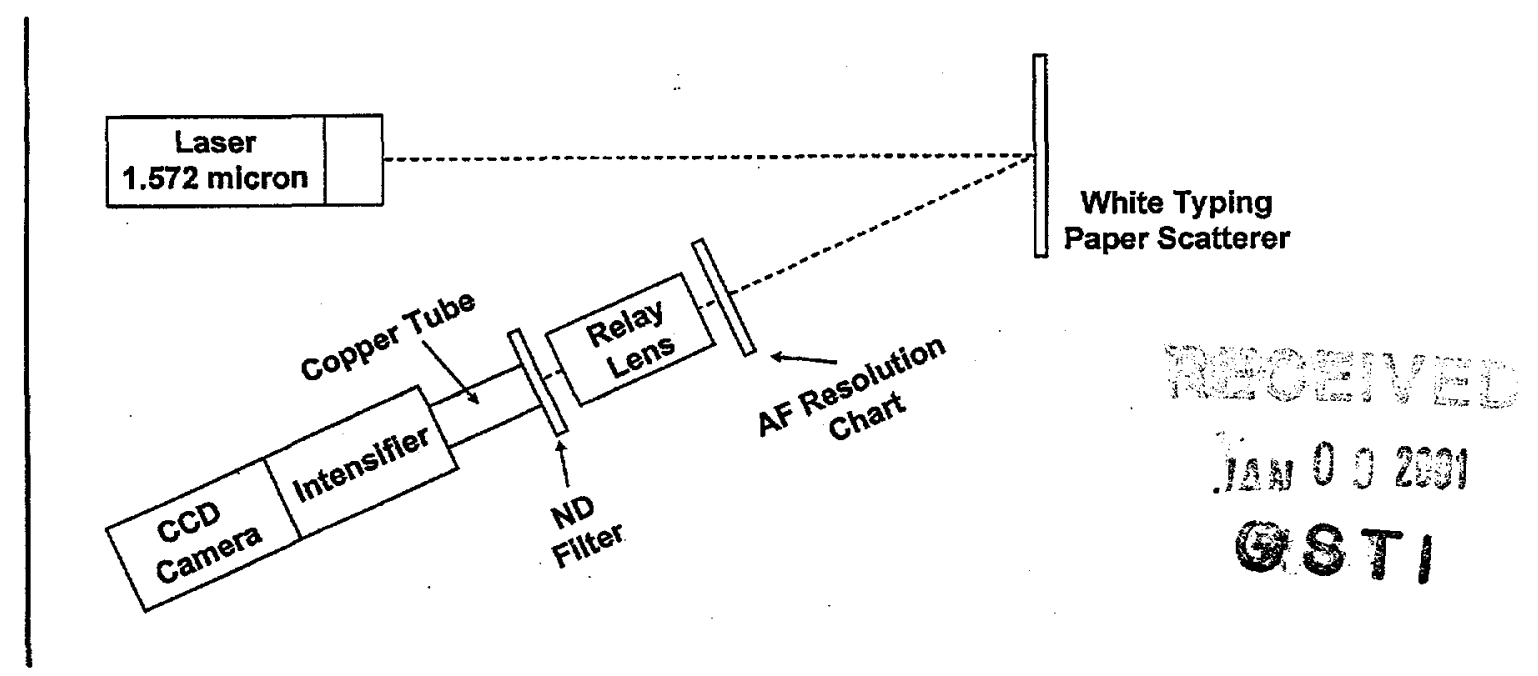

Figure 1. Initial experiment setup to measure the Intevac intensifier resolution and sensitivity. The laser beam is scattered by the paper, producing a Lambertian source with characteristic $2 \pi$ emission geometry. The transparent/opaque pattern was positioned in the direct path of the reflected laser beam and imaged with a 1:1 magnification relay lens. The copper tube is used to shield the intensifier from scattered light arriving at the photocathode from paths other than through the relay lens. The "intensifier" in the schematic is actually two stages, with the first stage being the Intevac intensifier which is fiber coupled to a Proxitronic diode intensifier which is then fiber coupled to the CCD camera.

The Black Body measurements for the Intevac intensifier indicated threshold signals from temperatures in the vicinity of $623 \mathrm{~K}$ corresponding roughly to wavelengths in the 1-2 micron range which comprise the Intevac intensifier's spectral range. Using Planck's expression/equation for relationship between temperature and wavelength (ref 10), at $623 \mathrm{~K}$, the calculated integrated power between $800 \mathrm{~nm}$ and 1800 $\mathrm{nm}$ at the Black Body source plane is $1.23 \mathrm{~mW}$. With the experiment's solid angle collection efficiency of $0.09 \%$ and a $1 \mu$ s gate used for the Intevac intensifier, the effective flux energy incident on the photocathode was calculated as $1.1 \mathrm{pJ} \mathrm{cm}^{-2}$. The gate was a $6 \mathrm{kV}$ pulse on the intensifier's phosphor and the Schottky barrier voltage used was $4.0 \mathrm{~V}$. 


\section{COMPARISONS BETWEEN INTEVAC AND OPO/OPA SENSORS}

These sets of experiments were to measure the relative performances of the TEPC technology with that of the OPO/OPA system. The test facility allowed us to gain experience in transporting reflectance images sizable distances, including through atmospheric clutter.

The experimental setup is shown in figure 2. The key components are the "seed" laser which uses 1.064 micron light from a Nd:YAG to pump a deuterium Raman cell which converts the 1.064 micron light to 1.56 microns. A prism is used to separate the two components, directing the 1.56 micron beam through various optics, filters, expanders, etc., to produce a "probe" or "broadcast" laser pulse. This beam is routed through a cloud chamber and onto a model airplane (Boeing 737) to provide a "reflectance" image that will be transported approximately 30 meters, via a second pass through the cloud chamber, onto an Lithium triborate (LBO) non-linear optical crystal. For characterizing the Intevac intensifier, the LBO crystal transmits the 1.56 micron beam through a set of neutral density filters and onto the intensifier's photocathode. Some light at near $780 \mathrm{~nm}$ from SHG in the crystal, although several orders of magnitude weaker, also reaches the intensifier. For the OPO/OPA system, the LBO crystal is pumped by a second laser at $532 \mathrm{~nm}$ causing wavelength conversion of the incident 1.56 micron beam to $807 \mathrm{~nm}$, with optical gain. This wavelength was imaged onto a Silicon Mountain Design CCD camera.

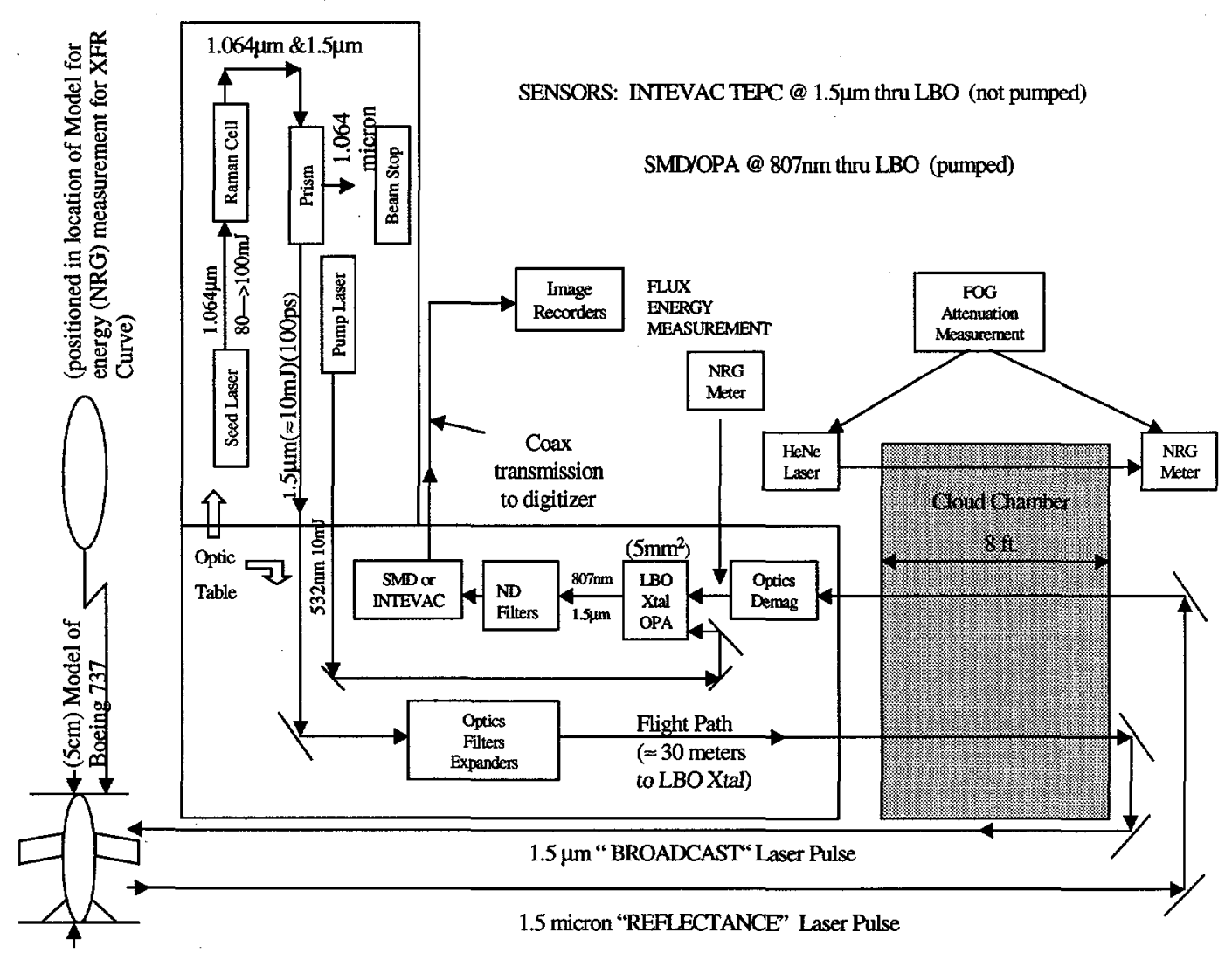

Figure 2. The reflectance imaging and optical setup for transporting 1.56 micron images (labeled 1.5 microns in the illustration) of a model Boeing 737 airplane through a controlled optical density cloud/fog chamber onto an LBO crystal. Following the crystal are either the Intevac intensifier or SMD CCD camera. 
The dynamic range transfer curve for the Intevac intensifier was generated first, using a mirror in place of the model airplane to provide a specular reflectance image, conserving the incident "broadcast" laser beam's coherent geometry, thereby providing a much brighter beam at the LBO crystal than that from the diffuse beam from the model airplane, which is basically a Lambertian source that suffers $1 / R^{2}$ losses consistent with the solid angle of the system. This was necessary in order to have high enough flux at the LBO crystal to measure absolute energy (labeled NRG in figure 2) with a $1 \mathrm{~cm}^{2}$ aperture area of the Laser Precision model RJ7620 radiometer. Using fluorescence cards to view the invisible beam we determined that the laser beam just filled or slightly under filled the aperture, so the measured values did not necessarily accurately define the flux per unit area. The laser flux was then attenuated with a range of neutral density (ND) filters to generate the transfer curve for the intensifier.

The images from the mirror are shown in figure 3. A starting laser flux of approximately $0.5 \mathrm{~mJ} / \mathrm{cm}^{2}$ was attenuated to generate the transfer curve. The top quad of images shows the saturation region of operation for the Intevac intensifier. This is the region where "electron starvation" (ref 11) occurs for the TE photocathode. The bottom quad of images corresponds to the intensifier's linear region of operation where signal brightness is proportional to input illumination levels. The amplitude data from these images are plotted in figure 4, depicting the entire transfer curve for the Intevac intensifier.

The inadequacy of the TE photocathode in terms of depletion or exhaustion of photoelectrons from overillumination is evident from the top quad of images shown in figure 3 . In those areas of over-illumination, instead of the image being "whiter" (corresponding to brighter), the image is darker. As noted above, this is referred to as "electron starvation" of the photocathode for TE devices, and results in the two regions for the transfer curve shown in figure 4. The flux magnitude where "electron starvation" ceases and linear operation starts is at approximately $50 \mathrm{~nJ} / \mathrm{cm}^{2}$ as noted for the images of figure 3 taken at ND 4 (saturated) and ND 5 (linear). The illumination which corresponds to the measured flux is the spot near the center for these images. As noted earlier, the other regions of illumination of the photocathode are from stray reflections reaching the intensifier.

A further examination of the "electron starvation" effect is illustrated in figure 5. For these tests, the input illumination was held constant and the Schottky barrier voltage was varied from 1 to 4 volts. The results for 2.0, 2.5,3.0, and 4.0 volts are shown in figure 5. The flux was estimated to be approximately $50 \mathrm{~nJ} / \mathrm{cm}^{2}$, but the apparent area from these images seems to be much smaller than $1 \mathrm{~cm}$, on the order of $10 \%$ of the area of the $25-\mathrm{mm}$ diameter photocathode. The experiment setup is being reviewed to estimate actual area of illumination at the time of this writing.

\section{NORMALIZATION OF AIRPLANE AND MIRROR IMAGES}

As noted above, the images recorded by the Intevac intensifier, shown in figure 6, (and also images recorded by the OPO/OPA sensors) for the model airplane were too weak to be measured with the radiometer. Therefore, the diffuse model airplane target was replaced with a mirror to provide a much brighter image for measuring flux magnitude. In order to get an estimate for the flux associated with the image of the airplane, the image was first digitized and recorded. Then, several rows (196 to 217) in the CCD signal corresponding to intensity profiles through the center of the airplane image were averaged and the signal in amplitude digital units (ADUs) was obtained. Next, one of the digitized mirror images was selected and the similar set of CCD rows (196 to 218) were averaged and their amplitude quantified. These data are shown in figure 7. The measured flux of approximately $43 \mathrm{~nJ} / \mathrm{cm}^{2}$ from the mirror image gave signal of $2596 \mathrm{ADU}$ counts (peak minus background). The airplane image gave a signal of $2114 \mathrm{ADU}$ counts, which when compared with the 2596 ADU counts gives approximately $35 \mathrm{~nJ} / \mathrm{cm}^{2}$ for the estimated flux for this image. Similarly measured, the flux for the OPO/OPA system was estimated to be approximately 25 to $50 \mathrm{~nJ} / \mathrm{cm}^{2}$ for the airplane image.

Images from both sensors were compiled into a single presentation, which is shown in figure 8. The images seem comparable in that figure, although the Intevac intensifier images are somewhat "fuzzier", which is attributed to image processing in the software used for the composite. The Intevac intensifier images are clearly sharper as observed in the earlier figures 6 and 7 . 


\section{INTEVAC TEPC DATA}

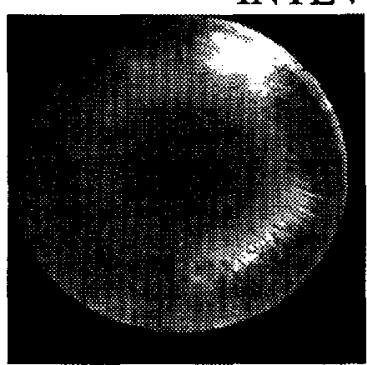

ND 3.0

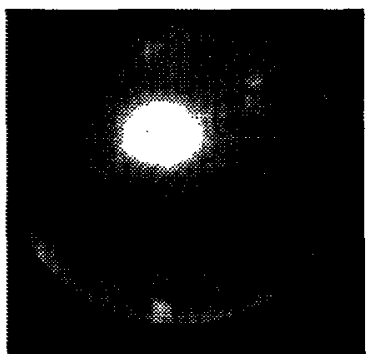

ND 4.0

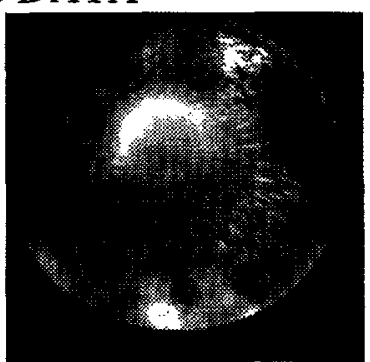

ND 3.5

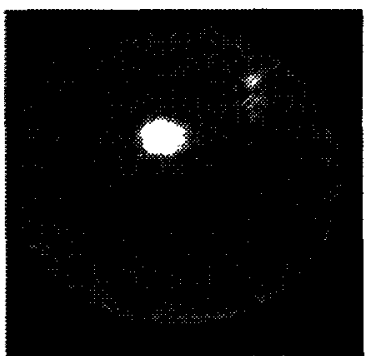

ND 5.0

Saturation Portion of Intevac XFR Curve

Laser Flux : 0.5 millijoules @ 1.5 micron, SBV: 3V, HV: 5kV

INTEVAC TEPC DATA

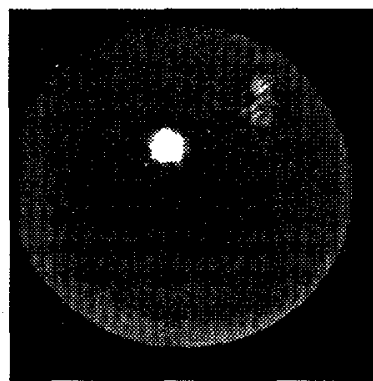

ND 6.0

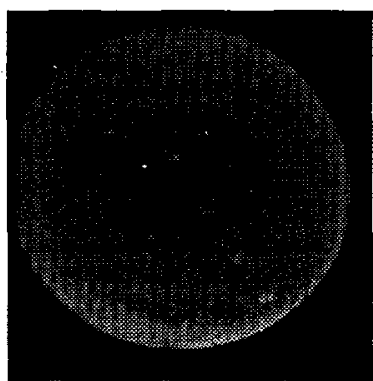

ND 8.0

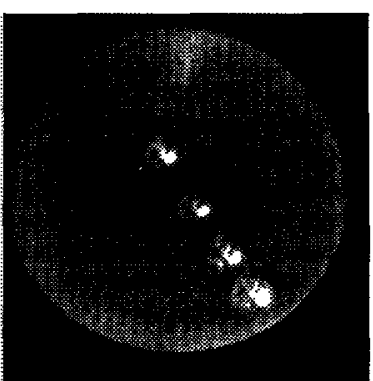

ND 7.0

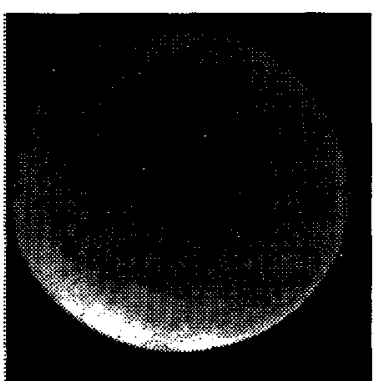

BG

Linear Portion of Intevac XFR Curve

Laser Flux: 0.5 millijoule @ 1.5 micron, SBV: 3V, HV: $5 \mathrm{kV}$

Figure 3. Image files showing performance of the Intevac intensifier's TE photocathode. The top quad of images shows the saturation region of transfer curve, and the bottom quad shows the linear region. 


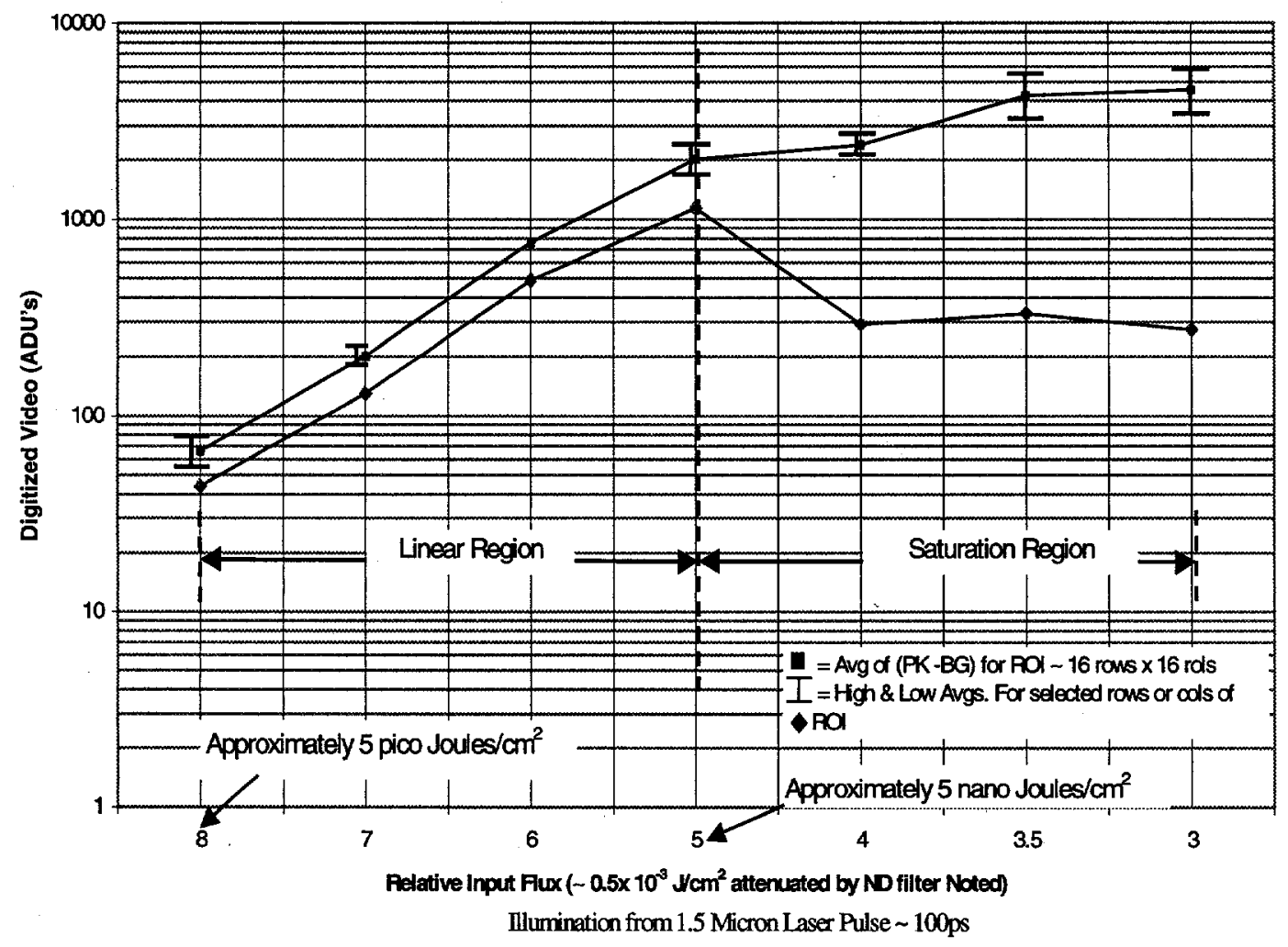

Figure 4. Dynamic range curve for Intevac intensifier from amplitude data of figure 3 images.

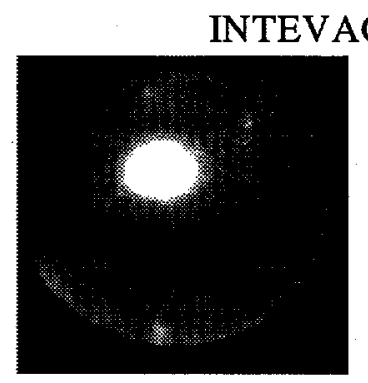

SBV $3.5 \mathrm{~V}$

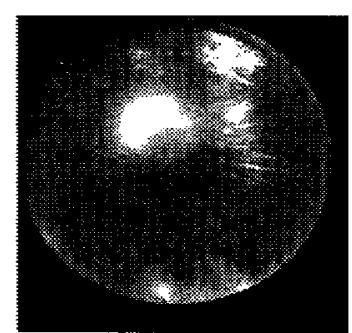

SBV 2.5V

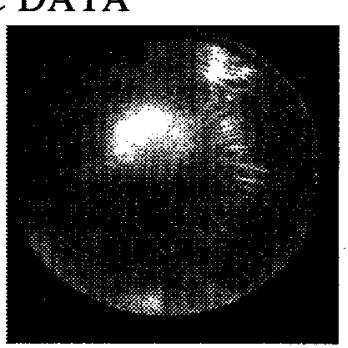

SBV 3V

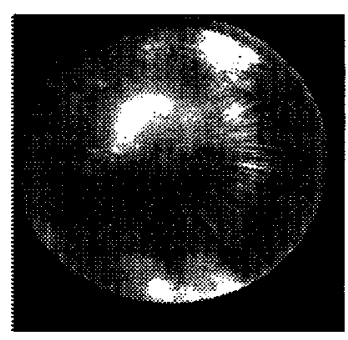

SBV 2V

Electron Starvation as Set of Schottky Barrier Voltage(SBV)

Laser Flux: 0.5 millijoules @ 1.5 micron, ND 4.0, HV: 5kV

Figure 5. Intevac "electron starvation" data for various Schottky voltages and constant illumination. 
INTEVAC TEPC DATA

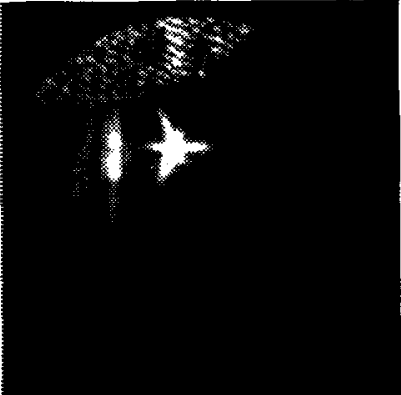

ND 0.0 No Scatter

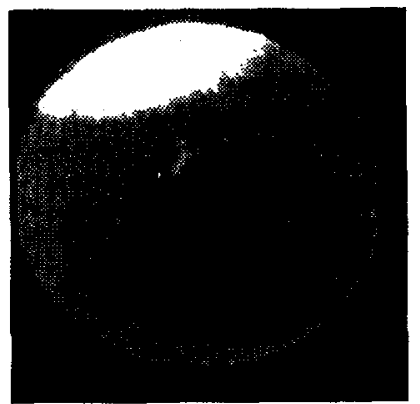

OD 2.0 (cloud chamber OD 1.0 laser passes through cloud chamber twice)

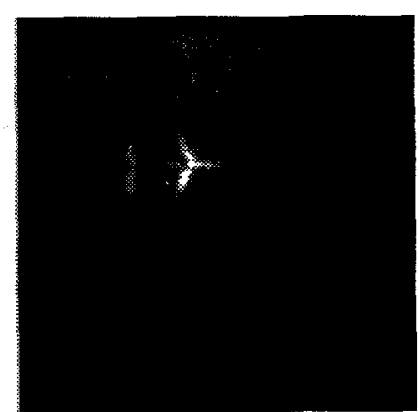

ND 1.0 No Scatter

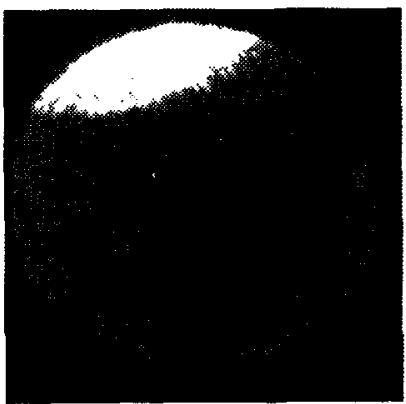

OD 3.0 (cloud chamber OD 1.5 laser passes through cloud chamber twice)

Reflective Images in Cloud Chamber

Figure 6. Images of model airplane for Intevac intensifier from variable optical density attenuation in the cloud chamber. The bright regions of images near the top are due to unintentional stray light.

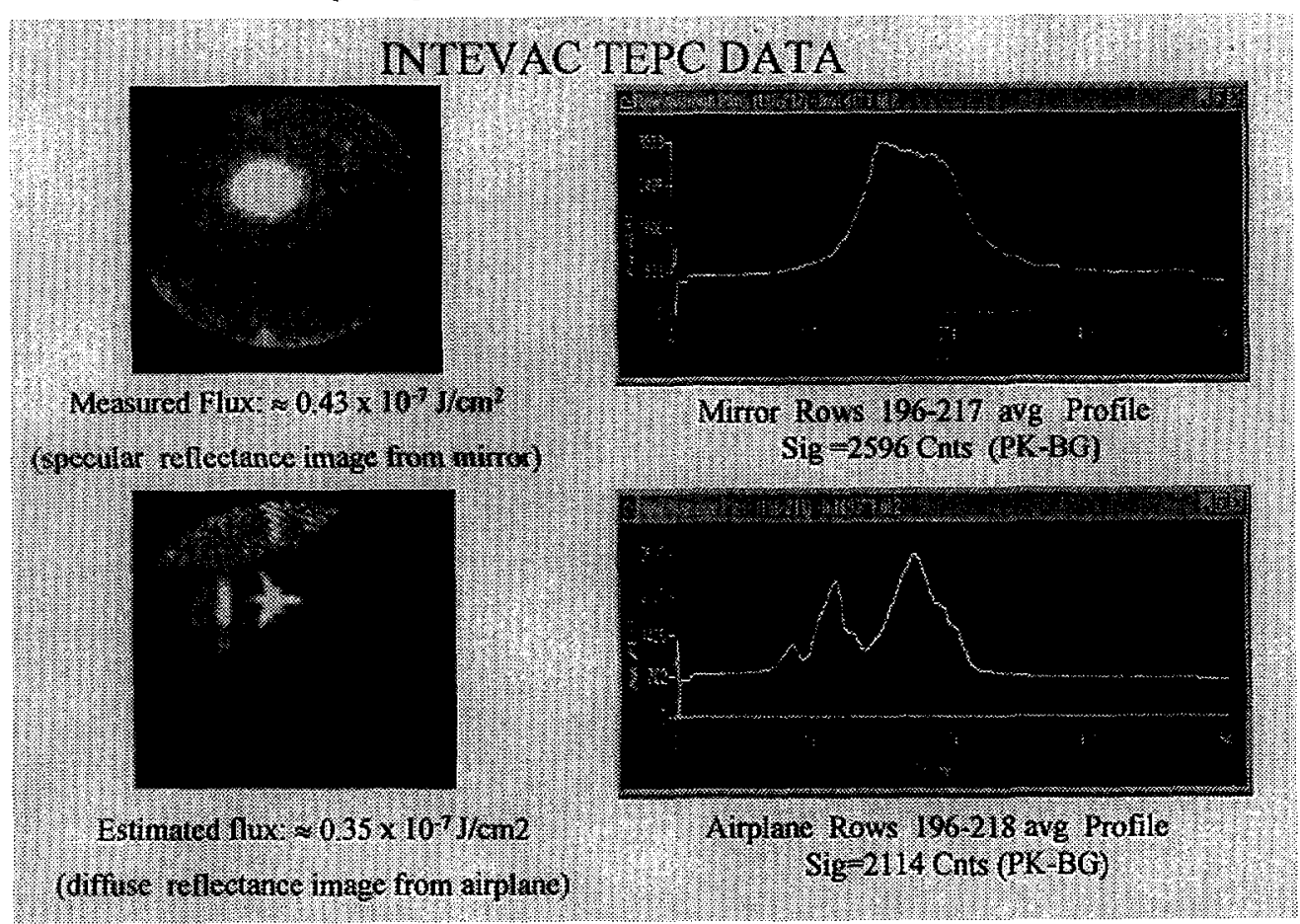

Figure 7. Normalization of model airplane and mirror images to obtain estimate of flux incident on the Intevac intensifier for the above airplane image. This image corresponds to the ND 0.0 image of figure 6 . 


\section{Double Pass Cloud Attenuation}

OD 0

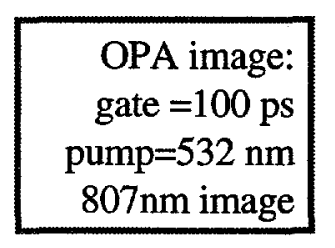

Intevac image:

gate $=10 \mu \mathrm{s}$

$1.56 \mu \mathrm{m}$ image
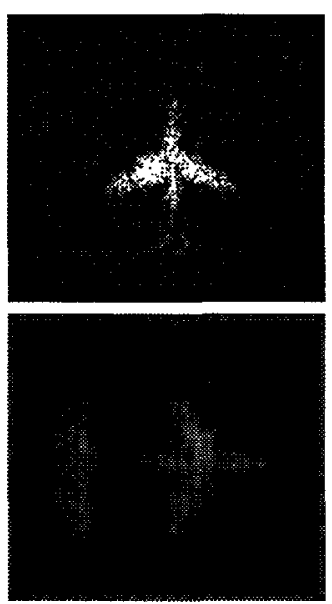

OD 2
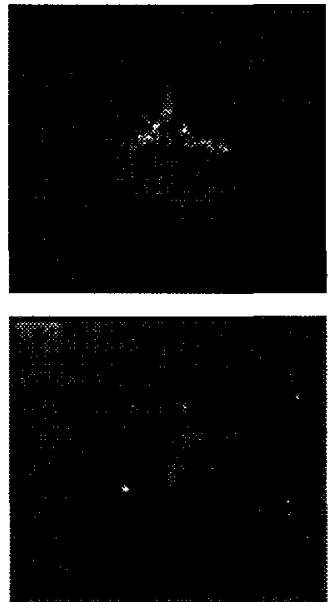

Broadcast: $\lambda=1.56 \mu \mathrm{m}, E=10 \mathrm{~mJ}, \quad$ Pump: $\lambda=532 \mathrm{~nm}, E=18 \mathrm{~mJ}$,

Figure 8. Comparison slides between Intevac "transferred electron" photocathode intensifier and nonlinear crystal OPO/OPA imaging technologies. The data were taken utilizing the cloud chamber shown earlier in figure 2. The OD 0 is with no scattering and the OD 2 is for two passes through the chamber as illustrated in figure 2. The short gate time for the OPO/OPA is dependent upon the lasers used. The noted gate duration of $10 \mu \mathrm{s}$ for the Intevac is not significant in terms of being any measure of it's sensitivity, since the laser pulse transporting the image was on the order of $100 \mathrm{ps}$. The gate was selected to allow for phosphor persistence decay time in order to collect all of it's light from a single excitation of the photocathode. If an ultra-fast decay phosphor were incorporated into the Intevac, and coupled to a GY-11 camera's stripline image intensifier, 100ps gates would also be possible.

\section{DISCUSSION OF TEST RESULTS}

Both approaches of providing capabilities for NIR and IR imaging appear to be possible solutions to the problem of "eye-safe" LADAR imaging to meet military requirements. The TE photocathode intensifier technology, although considered extremely attractive for this application, is regarded, by the authors, as proprietary technology to Intevac. Commercial availability of the technology is essential for successful incorporation into an IR or NIR LADAR imaging system. In addition, for high range resolution IR imaging, a sub-nanosecond scale time gate would be required. The TE photocathode "front end" would have to be combined with a stripline geometry similar to that in the GY-11 intensifier. Therefore, some mix of the non-linear crystal technology, utilizing the crystals either (1) only for up-conversion of IR wavelengths to visible wavelengths, coupled with a fast gated visible-range image intensifier for readout with a CCD camera such as the GY-11 system, or (2) for up-conversion, gating, and gain (the OPO/OPA configuration) for readout with a non-intensified CCD camera, appear to provide reasonable alternatives.

An extension of this study is underway to evaluate the merits of a hybrid system comprised of using an OPO/OPA in conjunction with the fast gated stripline intensifier of the GY-11 camera. Tradeoffs between optical gain in the OPO/OPA and electronic gain in the intensifier will be evaluated in the context of system performance, fielding complexity, and cost. 


\section{ACKNOWLEDGEMENTS}

The authors acknowledge technical support during experiments from Steven Jaramillo and Robert Gallegos of Los Alamos National Laboratory and from Wesley Helgeson and Steve Tucker of Sandia National Laboratories. We also acknowledge support from George Morgan of Los Alamos National Laboratory in the Black Body temperature to wavelength calculations. We also acknowledge valuable professional discussions with Paul Zagarino of Sharpenit Inc., Goleta, CA. This work was supported by the United States Department of Energy under Contract DE-AC04-94AL85000. Sandia is a multiprogram laboratory operated by Sandia Corporation, a Lockheed Martin Company, for the United states Department of Energy.

\section{REFERENCES}

1. G.J. Yates, et al, "High Speed Optical Shutter Coupled to Fast Readout CCD Camera," SPIE Conference on Sensors, Cameras, and Systems for Scientific/Industrial Applications, San Jose, Ca., January 25-26, 1999, SPIE Vol. 3649, pp. 22-30.

2. N.S.P. King, et al, "Underwater Mine Detection Utilizing Gated Intensifier Shutters Synchronized with Laser Reflectance Images from Submersed Targets," International Advanced Studies Institute (ISIA) Conference on Detection and Analysis of Subsurface Objects and Phenomena, High Speed Imaging Techniques and Applications, Naval Postgraduate School, Monterey, Ca., October 19-23, 1998, in Conference Proceedings.

3. T.E. McDonald, Jr., et-al, "Range Gated Imaging Experiments Using Gated Intensifiers," SPIE Conference on High-Speed Imaging and Sequence Analysis, San Jose, Ca., January 28-29, 1999, SPIE Vol. 3642, pp. 142-148.

4. K. Costello, et-al, " Transferred Electron Photocathode With Greater Than 5\% Quantum Efficiency Beyond 1 Micron," SPIE Conference on Electron Image Tubes and Image Intensifiers, San Jose, Ca., February 28-March 1, 1991, SPIE Vol. 1449, pp. 40-50.

5. V.W. Aebi, et-al, "Photocathode Development For A 1300-nm Streak Tube," SPIE Conference on Photodetectors and Power Meters, San Diego, Ca., July 15-16, 1993, SPIE Vol. 2022.

6. S.M. Cameron, D.E. Bliss, M.W. Kimmel, D.R. Neal, U.S. Patent No. 5936739 (10 August 1999).

7. T.E. McDonald, et-al, "Using Optical Parametric Oscillators (OPO) for Wavelength Shifting IR Images to Visible Spectrum," International Advanced Studies Institute (IASI) Symposium on Detection and Analysis of Sub-Surface Objects and Phenomena, High Speed Imaging Techniques and Applications, Monterey, Ca., October 19-23, 1998, in Conference Proceedings.

8. S.M. Cameron, et-al, "Remote Optical Imagery of Obscured Objects in Low-Visibility Environments Using Parametric Amplification," Meeting of the IRIS Specialty Group on Active Systems, Sandia National Laboratories, Albuquerque, NM, March 1998.

9. G.J. Yates, et-al, " Range-Gated LADAR Coherent Imaging Using Up-Conversion of IR-to-NIR Light For Imaging With a Visible-Range Fast Shuttered Intensified Digital CCD Camera," to be presented at IS\&T/SPIE Photonics West 2001-Electronic Imaging Symposium, High-Speed Imaging and Sequence Analysis III Conference, January 21-26, 2001, San Jose, Ca.

10. R.B. Leighton, editor, "Principles of Modern Physics," McGraw-Hill Book Co., Inc., copyright 1959, equation (13) of section 2-1, page 65.

11. K. Costello, Intevac, personal communication, May-July, 2000. 